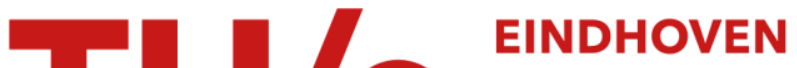 \\ UNIVERSITY OF \\ TECHNOLOGY
}

\section{Retrieving pulse profiles from pump-probe measurements on magnetization dynamics}

\section{Citation for published version (APA):}

Józsa, C., Rietjens, J. H. H., Kampen, van, M., Smalbrugge, E., Smit, M. K., Jonge, de, W. J. M., \& Koopmans, B. (2004). Retrieving pulse profiles from pump-probe measurements on magnetization dynamics. Journal of Applied Physics, 95(11), 7447-4. https://doi.org/10.1063/1.1667440

DOI:

$10.1063 / 1.1667440$

Document status and date:

Published: 01/01/2004

\section{Document Version:}

Publisher's PDF, also known as Version of Record (includes final page, issue and volume numbers)

\section{Please check the document version of this publication:}

- A submitted manuscript is the version of the article upon submission and before peer-review. There can be important differences between the submitted version and the official published version of record. People interested in the research are advised to contact the author for the final version of the publication, or visit the $\mathrm{DOI}$ to the publisher's website.

- The final author version and the galley proof are versions of the publication after peer review.

- The final published version features the final layout of the paper including the volume, issue and page numbers.

Link to publication

\section{General rights}

Copyright and moral rights for the publications made accessible in the public portal are retained by the authors and/or other copyright owners and it is a condition of accessing publications that users recognise and abide by the legal requirements associated with these rights.

- Users may download and print one copy of any publication from the public portal for the purpose of private study or research.

- You may not further distribute the material or use it for any profit-making activity or commercial gain

- You may freely distribute the URL identifying the publication in the public portal.

If the publication is distributed under the terms of Article 25fa of the Dutch Copyright Act, indicated by the "Taverne" license above, please follow below link for the End User Agreement:

www.tue.nl/taverne

Take down policy

If you believe that this document breaches copyright please contact us at:

openaccess@tue.nl

providing details and we will investigate your claim. 


\title{
Retrieving pulse profiles from pump-probe measurements on magnetization dynamics
}

\author{
C. Jozsa, ${ }^{\text {a) }}$ J. H. H. Rietjens, and M. van Kampen \\ Department of Applied Physics, Center for NanoMaterials and COBRA Research Institute, \\ Eindhoven University of Technology, P.O. Box 513, 5600 MB Eindhoven, The Netherlands \\ E. Smalbrugge and M. K. Smit \\ Department of Electrical Engineering, Center for NanoMaterials and COBRA Research Institute, \\ Eindhoven University of Technology, P.O. Box 513, 5600 MB Eindhoven, The Netherlands
}

W. J. M. de Jonge and B. Koopmans

Department of Applied Physics, Center for NanoMaterials and COBRA Research Institute, Eindhoven University of Technology, P.O. Box 513, 5600 MB Eindhoven, The Netherlands

(Presented on 9 January 2004)

\begin{abstract}
A method for back-tracing magnetic field pulses in pump-probe-type magnetization dynamics measurements is presented. Solving vectorially the Landau-Lifshitz-Gilbert equation for our field-induced measurement geometry yields field pulse profiles fulfilling the theoretical expectations on a $100 \mathrm{ps}$ timescale. Applying the method to our earlier, all-optical-type pump-probe measurements, the claim that the optical pulse triggers an ultrafast anisotropy field pulse gets a direct proof: we derive a pulse consisting of a delta-peak type, full width at half maximum $<5 \mathrm{ps}$ impulse on top of a very fast rising step-like profile. Application of the method to other, less trivial pump-probe schemes can contribute to the development of novel type magnetic recording technologies. (C) 2004 American Institute of Physics. [DOI: 10.1063/1.1667440]
\end{abstract}

Studies of fast (subnanosecond) magnetization phenomena with a diffraction limited spatial resolution are nowadays possible via pump-probe-type magneto-optical measurements. ${ }^{1-5}$ Using femtosecond pulsed lasers in a stroboscopic way, one can explore the temporal evolution of the local magnetization of thin layers and microstructures as a vectorial quantity reacting to external influences such as intense short heat pulses, ${ }^{2}$ fast pulsed magnetic fields,${ }^{5,6}$ or a combination thereof.

At the same time, the needs of the current recording technology push our interest toward studying nanosecond magnetic and magneto-optic phenomena of confined structures. It has become clear that achieving stable switching conditions in the precessional regime, requires fine tuning of the field pulses to a high degree of precision. ${ }^{3-7}$ Therefore, an important problem is the characterization (amplitude, rise/ fall time, duration, flatness, etc.) of the magnetic field pulse itself at the position of the micromagnetic entity under consideration.

Our approach is separating the Landau-Lifshitz-Gilbert (LLG) equation of magnetization motion into its vectorial components and applying the obtained system onto different experimental data. In this article, we present the back-tracing of field pulses in the case of our recent vectorial measurements on field-induced precessional dynamics and earlier alloptical studies on gigahertz precession. ${ }^{2}$ In principle, there is no direct obstacle for extending the model and calculus to more complex cases.

\footnotetext{
a)Electronic address: c.jozsa@tue.nl
}

A pump-probe-type setup based on the measurement of the magneto-optical Kerr effect (MOKE) versus time is shown in Fig. 1. Each pump pulse (magnetic or laser pulse) is followed by a second, weak probing laser pulse. The probe beam has a well-defined incident polarization that can be compared to the final polarization of the beam after being reflected from the sample surface. The time difference ("delay time") between the arrival of the pump and probe pulses is controlled during the experiment with a mechanical delay line.

In our studies on field-induced precessional dynamics, we use as pump source magnetic field pulses generated by current pulses running through a microscopic coplanar waveguide structure $(200 \mathrm{~nm}$ thick, $50 \mu \mathrm{m}$ wide $\mathrm{Au}$ stripes on GaAs). The waveguide is electrically coupled to a commercial pulsegenerator (rise/fall times of typically $120 \mathrm{ps)}$ ). The pulse generator is triggered by the monitor signal of the built-in photodiode of a mode-locked Ti:Sa laser $(80 \mathrm{MHz}$, $100 \mathrm{fs}$ pulses at $780 \mathrm{~nm}, 0.05 \mathrm{~nJ} /$ pulse). The electrical pul-

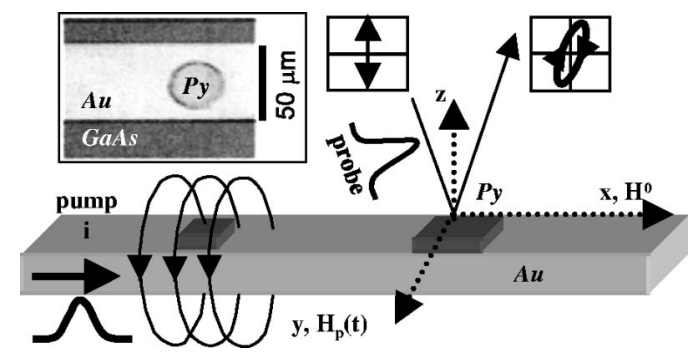

FIG. 1. Experimental pump-probe setup. Top left inset: microscopical photograph of an actual sample, top view. 
segenerator is switched on and off by an external $10 \mathrm{kHz}$ square wave, that is also used to trigger the lock-in amplifier. The MOKE signal measured by a polarizer/analyzer combination, can be linearly transformed into magnetization units.

The waveguide structure was covered by an electrically insulating $\mathrm{SiN}_{x}$ layer, prior to sputter deposition of a $30 \mathrm{~nm}$ Permalloy (Py) layer. Discs of 4 to $50 \mu \mathrm{m}$ diameter were structured by a lift-off technique. A photograph of a microscopic sample is shown as an inset in Fig. 1. Field pulses at an amplitude up to $350 \mathrm{~A} / \mathrm{m}$ are used; this value is well below the coercivity of our Py "dots" $(650 \mathrm{~A} / \mathrm{m})$. Therefore, we cannot achieve switching of magnetization during these measurements.

Different methods have been reported recently for simultaneous measurements of multiple components of the magnetization vector. ${ }^{5,6}$ We use a vectorial scheme as follows. A laser objective (NA 0.38) focuses a wide, aperture-filling perpendicular laser beam on the sample. Since there is a $15^{\circ}$ difference between the angle of incidence of the top-bottom $(A, C)$, respectively left-right $(B, D)$ regions of the beam, they probe different ratios of the three components of the magnetization vector. Using a four-quadrant photodetector, these four sections of the reflected laser beam are measured with independent lock-in amplifiers. After normalization to the dc intensities, we can see that $M_{z} \propto(\mathrm{A}+\mathrm{B}+\mathrm{C}+\mathrm{D}), M_{y} \propto(\mathrm{A}$ $+\mathrm{B}-\mathrm{C}-\mathrm{D})$, and $M_{x} \propto(\mathrm{A}-\mathrm{B}+\mathrm{C}-\mathrm{D})$. In the present experiments, we use the setup simplified to two quadrants $(\mathrm{A}, \mathrm{C})$ and two components $\left(M_{y}, M_{z}\right)$.

The all-optical experimental setup uses, instead of magnetic field pulses, intense ultrashort laser pulses combined with a double-modulation measurement technique described in detail in Ref. 2. Measurements were done on a large variety of (mostly unstructured) thin layers of ferromagnetic metals; the results presented here were obtained on a polycrystalline, $7 \mathrm{~nm}$ thin $\mathrm{Ni}$ layer on a $\mathrm{Si} / \mathrm{SiO}_{2}$ substrate with an in-plane easy axis for the magnetization vector.

Next, we present an analysis used to trace back the applied field pulses (electrically or optically induced ones) from the observed precessional motion of $\vec{M}$. The dynamics is described by the LLG equation

$$
\frac{d \vec{M}(t)}{d t}=-\gamma \mu_{0}\left(\vec{M} \times \vec{H}_{\mathrm{eff}}\right)+\frac{\alpha}{M_{s}}\left(\vec{M} \times \frac{d \vec{M}(t)}{d t}\right),
$$

where $\gamma$ is the gyromagnetic ratio, $\alpha$ is the (dimensionless) damping parameter, and $M_{s}$ is the saturation magnetization of the magnetic layer.

In the case of our field-induced magnetization measurements, we have the pulse field $\vec{H}_{p}(t) \equiv H_{p}(t) \cdot \hat{y}$ and the bias field $\vec{H}_{d c} \equiv H_{0} \cdot \hat{x}$ both in the film plane $(x y)$, and perpendicular to each other. The demagnetization field is oriented opposite to the $M_{z}$ component of the magnetization and equal in amplitude with it, $\vec{H}_{\text {demag }} \equiv M_{z} \cdot \hat{z}$. We also assume a weak perturbation, thus, $M_{x}=$ const $=M_{s}$. With these considerations, Eq. (1) can be separated for the three directions and simplified to the following form:

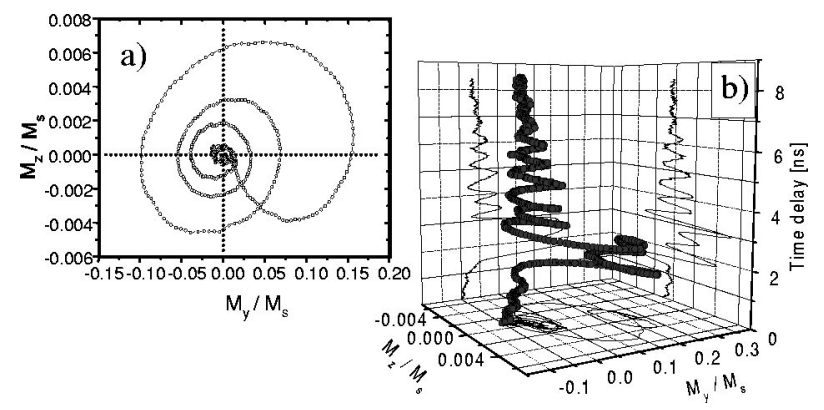

FIG. 2. Vectorial measurement of field-induced precessional dynamics. (a) $0.5 \mathrm{~ns}$ (short) field pulse, $M_{y}-M_{z}$ plot. (b) $1.5 \mathrm{~ns}$ (long) field pulse, $M_{y}$ $-M_{z}-\Delta t$ plot. Magnetization values are normalized to $M_{s}$. In both cases, an external DC field of $2.4 \mathrm{kA} / \mathrm{m}$ is applied along the $x$-axis.

$$
\begin{aligned}
& \dot{M}_{x}=0, \\
& \dot{M}_{y}=-\gamma \mu_{0}\left(M_{s}+H_{0}\right) M_{z}-\alpha \dot{M}_{z}, \\
& \dot{M}_{z}=-\gamma \mu_{0}\left(M_{s} \cdot H_{p}(t)-M_{y} H_{0}\right)+\alpha \dot{M}_{y} .
\end{aligned}
$$

The goal is to determine from these equations the field pulse $H_{p}(t)$ when measuring one of the two components $\left(M_{z}\right.$ or $M_{y}$ ). Solving Eq. (2) for $H_{p}$ yields

$$
\begin{aligned}
H_{p}(t)= & -\frac{\alpha^{2}+1}{\gamma \mu_{0}} \dot{m}_{z}-\alpha\left(M_{s}+2 H_{0}\right) m_{z} \\
& -\gamma \mu_{0} H_{0}\left(M_{s}+H_{0}\right) \int_{-\infty}^{t} m_{z} d t
\end{aligned}
$$

for the case of measuring $M_{z}$, and

$$
\begin{aligned}
H_{p}(t)= & -\frac{\alpha^{2}+1}{\gamma^{2} \mu_{0}^{2}\left(M_{s}+H_{0}\right)} \ddot{m}_{y}+\frac{\alpha\left(M_{s}+2 H_{0}\right)}{\gamma \mu_{0}\left(M_{s}+H_{0}\right)} \dot{m}_{y} \\
& +H_{0} \cdot m_{y}
\end{aligned}
$$

for the case of measuring $M_{y}$, with $M_{i}=m_{i} \cdot M_{s}$.

The geometry of the all-optical measurements is slightly different. In particular, $\vec{H}_{p}$ is oriented perpendicularly to the film plane, due to the temperature-dependence of the shape anisotropy: $\vec{H}_{p}(t)=H_{p}(t) \cdot \hat{z}$. This yields a slightly more complicated transformation, to be published in full detail elsewhere.

In all the above cases we can obtain a formula for the field pulse that contains only the measurement data, its derivatives and/or integrals, and some free parameters.

In Fig. 2, two measurements are shown with electrically induced field pulses of $\sim 500 \mathrm{ps}$ (a) and $\sim 1500 \mathrm{ps}$ (b), respectively. Typical results are in agreement with theoretical predictions, e.g., Ref. 8. In the case of the long pulse measurement [Fig. 2(b)], the precession starts to damp out after the first step (the rise of the field pulse). However, the magnetization does not reach the new equilibrium before the falling edge of the pulse exerts a new torque on it.

Applying Eqs. (3) and (4) to recover the original field pulse profile, necessitates double differentiation and integration of the measured datasets. In case of a real measurement with finite noise, the differentiation needs adequate smoothing of the data, while the integration induces a slope in the 

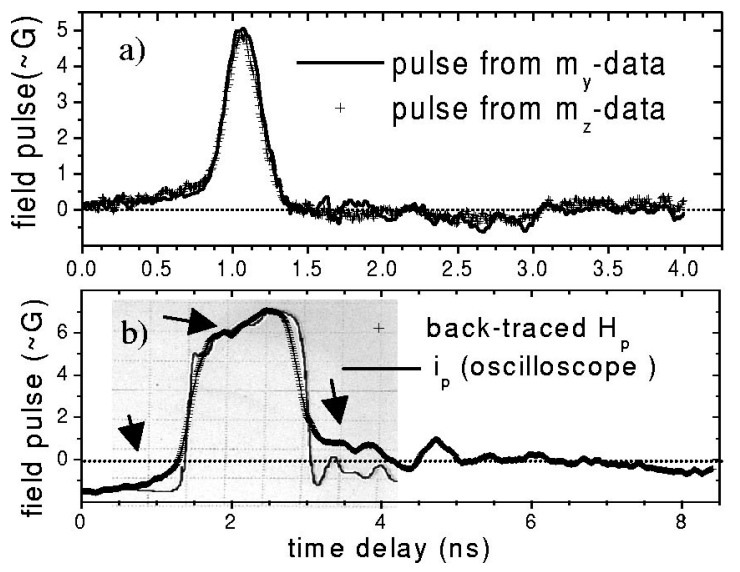

FIG. 3. Short (a) and long (b) field pulses back-calculated from the measurements shown in Fig. 2. Note the timescale difference between (a) and (b). The long-pulse measurement on (b) is overlapped with an image of an oscilloscope display showing the $1.5 \mathrm{~ns}$ electric pulse, on the same timescale.

dataset whenever there is an inaccuracy in centering the precessional data around zero. Taking care of these two problems, very acceptable results are obtained for short and long field pulses (Fig. 3). In Fig. 3(a) the profiles derived from the two independent magnetization components almost completely overlap, illustrating that the procedure yields unambiguous results. As a further comparison, we paste in a screen capture of a fast oscilloscope showing the electrical pulse entering an ideal $50-\Omega$ impedance matched probe [Fig. 3(b)]. The arrows point to fine pulse details shorter than the time scale of the oscillation period, that are similar on both long pulse images, indicating the high accuracy of the method. The amplitude of the obtained field pulse $(350 \mathrm{~A} / \mathrm{m})$ also corresponds in order of magnitude to the values obtained through a finite element method simulation. A damping parameter of $\alpha=0.01$ can be deduced, in good agreement with other values obtained for thin Py layers. ${ }^{6}$

A typical all-optical polar measurement is shown in Fig. 4(a). Laser pulses of $\sim 100 \mathrm{fs}$ are used, and a bias field of $200 \mathrm{kA} / \mathrm{m}$ is applied at an angle of $35^{\circ}$ with respect to the

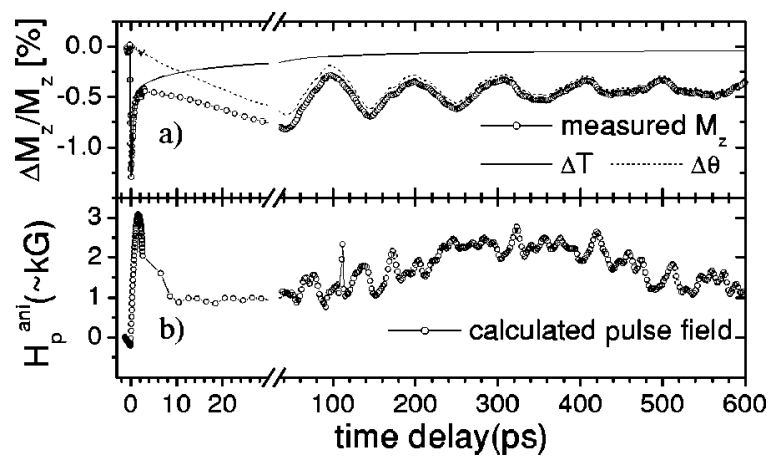

FIG. 4. All-optical-type measurement (a) and its back-calculated pulse field (b). surface normal to induce a canted orientation of $\vec{M}$, and assure a sufficient in-plane field as well. Due to the applied bias field that is considerably larger than in the presented field-induced measurements, the precession frequency is also higher: $\omega=\gamma \mu_{0} \sqrt{H_{0}\left(H_{0}+M_{s}\right)}=2 \pi \cdot 9.98 \mathrm{GHz}$.

In order to apply our back-tracing procedure, first the laser-induced reduction of the magnetic moment $(\Delta T$, solid line) has to be separated from the orientational effect $(\Delta \theta$, dashed line). Assuming that $\Delta M_{z}$ for $t<1 \mathrm{ps}$ is dominated by the first effect, and considering a slow recovery of the temperature of the $\mathrm{Ni}$ film after $1 \mathrm{ps}$, provides the dashed line as an estimate of the orientational contribution to the measurement. This is used as an input for deriving the effective pulse profile.

The earlier claim that the optical pulse triggers an ultrafast anisotropy field pulse ${ }^{2}$ is substantiated by the derived pulse profile shown in Fig. 4(b): an extremely fast delta-like pulse (full width at half maximum $<5 \mathrm{ps}$ ) superposed on a step-like background with a rise time $<5 \mathrm{ps}$. The derived pulse shape depends slightly on the exact procedure used to separate the amplitude and orientation of the transient magnetization. However, independent of those details, a deltalike pulse always appears, with a rather unique value for its integrated area. A completely unambiguous profile would be achieved when performing a vectorial measurement. Finally, we stress that similar behavior was observed on a large number of systems ( $\mathrm{Ni}$ and Py thin films, different substrates, film thicknesses, anisotropy, and applied fields) presented in Ref. 2.

In conclusion, our vectorial magnetization measurements depict a field-induced dynamics behavior that is in good agreement with the expectations. The calculus we describe was successfully applied to back-calculate the magnetic field pulses just above the strip line. The method is shown to work for more complicated cases as well, wherein the pulse profile cannot be determined by other means. Applied to our former all-optical experiments, the method demonstrates that the laser pulse triggers an ultrashort anisotropy field pulse. We suggest further applications to other, less trivial pump-probe schemes, such as multiple field pulses ${ }^{5}$ or field pulses combined with a laser heating pulse, of relevance for novel type technologies in magnetic recording.

The authors are grateful to J.J.M. Kwaspen, O. Kurnosikov, and J.T. Kohlhepp (TUE) for useful discussions and experimental support. This work is part of the research program of the "Stichting voor Fundamenteel Onderzoek der Materie (FOM)" which is financially supported by the "Nederlandse Organisatie voor Wetenschappelijk Onderzoek (NWO)."

\footnotetext{
${ }^{1}$ W. K. Hiebert et al., Phys. Rev. Lett. 79, 1134 (1997).

${ }^{2}$ M. van Kampen et al., Phys. Rev. Lett. 88, 227201 (2002).

${ }^{3}$ H. W. Schumacher et al., Phys. Rev. Lett. 90, 017204 (2003).

${ }^{4}$ H. W. Schumacher et al., Phys. Rev. Lett. 90, 017201 (2003).

${ }^{5}$ Th. Gerrits et al., Nature (London) 418, 509 (2002).

${ }^{6}$ W. K. Hiebert et al., Phys. Rev. B 68, 020402 (2003).

${ }^{7}$ S. Kaka et al., Appl. Phys. Lett. 80, 2958 (2002).

${ }^{8}$ L. He and W. D. Doyle, J. Appl. Phys. 79, 6489 (1996).
} 